\title{
OPTIMAL BOARDING POLICIES FOR THIN PASSENGERS
}

\author{
EITAN BACHMAT * AND \\ DANIEL BEREND, ${ }^{* *}$ Ben-Gurion University \\ LUBA SAPIR, ${ }^{* * *}$ Ben-Gurion University and Holon Institute of Technology \\ STEVEN SKIENA, ${ }^{* * * *}$ Stony Brook University
}

\begin{abstract}
We deal with the problem of seating an airplane's passengers optimally, namely in the fastest way. Under several simplifying assumptions, whereby the passengers are infinitely thin and react within a constant time to boarding announcements, we are able to rewrite the asymptotic problem as a calculus of variations problem with constraints. This problem is solved in turn using elementary methods. While the optimal policy is not unique, we identify a rigid discrete structure which is common to all solutions. We also compare the (nontrivial) optimal solutions we find with some simple boarding policies, one of which is shown to be near-optimal.
\end{abstract}

Keywords: Stochastic geometry; airplane boarding; optimality; optimal airplane boarding.

2000 Mathematics Subject Classification: Primary 49K45; 90B06

Secondary 90B22; 65K10

\section{Introduction}

The process of airplane boarding is experienced daily by millions of passengers worldwide. Airlines have adopted a variety of boarding strategies in the hope of reducing the gate turnaround time for airplanes. Significant reductions in gate delays would improve the quality of life for long-suffering air travelers, and yield significant economic benefits from more efficient use of aircraft and airport infrastructure; see [12], [14], and [16].

Despite the fact that the boarding process is an important part of the customers' flying experience, there has been little effort in the way of airplane boarding analysis; see [16]. The efforts [10], [12], [14]-[16] thus far have been simulation and heuristics based. To the best of our knowledge, no previous attempt at a rigorous mathematical analysis has been made.

The most pervasive strategy currently employed links boarding time to seat assignment. The airline specifies which rows may start boarding at any given time. The policy is implemented by announcements of the form 'passengers from rows 30 and above are now welcome to board the plane'. Such strategies are back-to-front boarding strategies, since they board passengers from

Received 13 February 2006; revision received 7 September 2007.

* Postal address: Department of Computer Science, Ben-Gurion University, Beer-Sheva, 84105, Israel.

Email address: ebachmat@cs.bgu.ac.il

** Postal address: Department of Mathematics and Department of Computer Science, Ben-Gurion University, BeerSheva, 84105, Israel. Email address: berend@cs.bgu.ac.il

*** Postal address: Department of Industrial Engineering and Management, Ben-Gurion University, Beer-Sheva, 84105, Israel. Email address: 1sapir@bgu.ac.il

**** Postal address: Department of Computer Science, Stony Brook University, Stony Brook, NY 11794-4400, USA. Email address: skiena@cs.sunysb.edu 
the back of the airplane first. Moreover, current policies may be considered to be jump policies in the sense that the announcements allow passengers in a certain group of contiguous rows to join the queue simultaneously. It is possible to consider other back-to-front policies which gradually allow passengers to join the boarding queue, one row at a time. Such policies may be implemented using a display at the terminal which shows which row (and those beyond it) may now join the queue. In jump policies the row numbers on the display will decrease by jumps, while in gradual policies they will steadily decrease by at most one at any given time. Among the gradual policies, we can single out the constant pace policies in which the displayed row number decreases by one after a constant time interval. When assessing boarding policies we also need to model the way in which passengers react to the policies. In this paper we assume that passengers join the boarding queue uniformly within a constant time interval of being allowed to do so by the policy. The size of the interval, which we denote by $\alpha$, is a reciprocal measure of the attentiveness of passengers. The rate at which passengers from new rows are allowed to join the boarding queue in the constant rate policy, and the size of jumps in jump policies, may be adjustable parameters, depending on how attentive the passengers are to the boarding policy. The optimal boarding policies and boarding times will also depend on $\alpha$.

In this paper we find a family, parameterized by $\alpha$, of asymptotically optimal back-to-front boarding policies under the assumption that passengers are infinitely thin or, equivalently, that airplanes are very spacious, i.e. the distance between successive rows in the airplane is very large. While this assumption may seem unrealistic, it is still instructive to consider this case since the obtained results are rather precise and the methods introduced to tackle it can be applied more generally. The problem is solved by reducing the problem of airplane boarding to the problem of finding the longest increasing subsequence among uniformly sampled points in a planar domain. The problem is then reduced to an isoperimetric type problem of finding an optimal domain.

The optimal policies we present are rather complex; see Section 4 for complete details. However, we can show that constant rate policies are near-optimal and are better than group policies, after the policy parameters are adjusted to the attentiveness of the passengers. The optimal policies are also not unique, but we identify an $\alpha$-dependent discrete substructure, common to all optimal solutions.

The general problem of airplane boarding for nonthin passengers is considered in a series of papers, including [6] and [7], which show that airplane boarding time can be reduced to the problem of computing the longest curve in a two-dimensional domain, equipped with a Lorentzian metric, which is used for modeling the boarding process. The boarding process itself is a discrete version of a wave front in the Lorentzian geometry. Some connect-the-dots type problems [2], surface growth processes [13], and disk scheduling problems can be modeled similarly [3], [4]. The Lorentzian metric which corresponds to the cases considered in this paper is flat (no curvature). The problem is thus easier to deal with and can be solved completely. The results for nonthin passengers [5], [6] turn out to be very different from the results for thin passengers. In fact, policies which are good for thin passengers are bad for nonthin passengers and vice versa. The assumption about passengers being thin or that airplanes are spacious is crucial.

The paper is organized as follows. In Section 2 we carefully present our model of the boarding process. We also show that increasing subsequences in a permutation, attached to the boarding process, correspond to sequences of passengers, each blocking his successor's way to his assigned seat. Thus, boarding time will coincide with the length of the maximal increasing subsequence. In Section 3 we digress to a discussion of the asymptotics of maximal increasing 
subsequences of permutations. The main results are stated in Section 4. Section 5 is devoted to the proofs of our results. In Section 6 we consider the examples of jump and constant rate policies and compare them with the optimal policies.

\section{The model of airplane boarding}

\subsection{The boarding process}

Let us describe our model of the airplane boarding process. We assume that passengers are assigned seats in the airplane in advance of the boarding process. For simplicity, we also assume that each row in the airplane is designed for a single passenger. The airplane has $S$ seats. We denote the total amount of time allotted for joining the queue by $Q$. We represent passengers by points $(x, y)$ in the plane, where $x$ is the row assigned to the passenger and $y$ is the time at which he joins the boarding queue. For convenience, we shall normalize both variables; $x$ is replaced by $x / S$ and $y$ by $y / Q$. Via these normalizations, the point $(x, y)$ will lie in the unit square. Passengers are assumed to be infinitely thin. We assume that boarding starts only after all passengers have joined the queue at time $Q$, and thus the queueing time $Q$ is not included in the boarding time. The reason for this choice is that we wish to compare different boarding policies, while queue delays such as a late arriving passenger are policy independent. As a result, only the order of passengers in the queue and their row numbers will affect the boarding process. Initially, all passengers line up in a queue in front of the airplane gate. Each passenger in the queue walks towards his or her designated row. Once passengers arrive at their designated seat, it takes them a fixed amount of time to get organized and clear the aisle by sitting down. This assumption leads to a synchronous boarding process, which we describe in terms of rounds. In the first round all passengers who can walk unobstructed all the way to their assigned row do so. Those who cannot reach their seat, owing to another passenger with a smaller row number obstructing their way, proceed as far as possible and queue behind an obstructing passenger. At the end of the round, all passengers who have reached their assigned row sit down simultaneously. Once the first round of passengers is seated, the remaining passengers advance forward again, beginning a second round of movement. The process repeats until everyone is seated. The number of rounds needed will be taken as our measure of the total boarding time.

We define a natural partial order on passengers. A passenger $A$ blocks another passenger $B$, and we denote $A \prec B$, if the latter may sit only after the former has done so. Formally, let $A=\left(x_{A}, y_{A}\right)$ and $B=\left(x_{B}, y_{B}\right)$. Then $A \prec B$ if $x_{A}<x_{B}$ and $y_{A}<y_{B}$. Intuitively, this condition means that $B$ arrives after $A$ and sits behind him. A chain in this partial order is an increasing sequence or an upright sequence.

In terms of the partial order, the boarding process is a well-known 'peeling' process, which can be traced to the work of Cantor on ordinal arithmetic. The process peels the partially ordered set by successively eliminating (in rounds) the minimal elements in the partial order. In our case the minimal elements are precisely the passengers which are unobstructed, and can thus proceed directly to their assigned seat. This shows that the two processes indeed coincide. We recall that a set $C$ in a partial order is a chain if every two elements $x, y \in C$ are comparable, i.e. either $x \prec y$ or $y \prec x$. A set $I$ is independent if no two elements of $I$ are comparable. The peeling process simultaneously provides a minimal decomposition of the poset into independent sets and a maximal chain in the poset. To see this, consider $R_{i}$, the set of passengers arriving at their seat during round $i$. By the definition of the peeling process, each $R_{i}$ is independent. To show that the $R_{i}$ s form a minimal decomposition into independent sets, 
we construct a chain, whose length equals the number of rounds, as follows. Each passenger (not seated in the first round) is assigned a pointer, which points to the last passenger who was responsible for blocking his or her way to the assigned row. This passenger obviously sat down in the previous round. Following the trail of pointers starting from a passenger who arrived at his seat in the last round, we identify a longest chain in the partial order. In particular, the number of rounds needed is the size of the longest chain in the partial order, or the longest increasing subsequence. The boarding process in fact coincides with patience sorting, a procedure which computes the longest increasing subsequence in a permutation. The corresponding permutation is obtained by indexing the passengers according to their $x$-coordinate, while the value of the permutation is the $y$-coordinate index. We refer the reader to [1] for a description of patience sorting and its importance in studying increasing subsequences.

\subsection{Airline policies}

An airline policy is represented by a function $L(x)$, which provides the earliest time at which a passenger from row $x$ may join the queue. Stated otherwise, the policy represented by $L$ allows passengers in row $x S$ to join the queue at time $y Q$. Back-to-front policies will obviously correspond to nonincreasing functions.

In addition to a boarding policy, we need a probabilistic model for the passenger's reaction to the boarding policy.

We consider the following reaction model, which we call the attentive reaction model. We assume that passengers follow the airline policy in the sense that a passenger in row $x$ does not join the queue before time $L(x)$. We also assume that there exists an attentiveness parameter $\alpha$, such that a passenger at row $x$ joins the queue uniformly within $\alpha$ time units of the earliest allowed boarding time $L(x)$. The parameter $\alpha$ is a reciprocal measure of the attentiveness of the passengers, with a small value of $\alpha$ corresponding to very attentive passengers. When considering policies $L$ in conjunction with this reaction model, we shall always assume that passengers are allowed at least $\alpha$ time units to board, or, equivalently, that $L(x) \leq 1-\alpha$. The policy $L$ coupled with the attentive reaction model with parameter $\alpha$ determine a density $p_{L, \alpha}$, which describes the probability of a passenger from row $x$ joining the queue at time $y$. More precisely, our assumption that passengers from row $x$ do not join the queue before time $L(x)$, but do join within $\alpha$ time units, means that $p_{L, \alpha}(x, y)=0$ for $y \leq L(x)$ and $y \geq L(x)+\alpha$. The assumption of uniform boarding within the time interval $L(x) \leq y \leq L(x)+\alpha$ means that $p_{L, \alpha}(x, y)=1 / \alpha$ for $L(x) \leq y \leq L(x)+\alpha$. Given some value of $\alpha$, it will sometimes be more convenient for us to represent a policy by the function $U=L+\alpha$ rather than by $L$ itself. The representation of a policy by $U$ is the upper representation. We will use both representations interchangeably, and thus $p_{L, \alpha}$ is the same as $p_{U, \alpha}$.

Following the discussion in Subsection 2.1, we may define the random variable $T_{L, \alpha, n}$, which represents the boarding time of $n$ passengers, given the policy $L$ and attentiveness parameter $\alpha$, as follows. We choose $n$ independent and identically distributed (i.i.d.) points from the unit square using the density $p_{L, \alpha}$. Here $T_{L, \alpha, n}$ denotes the length of the longest increasing subsequence among the $n$ points.

\section{The asymptotics of increasing subsequences}

The following result of [9] describes the asymptotic behavior of the maximal increasing subsequence of $n$ i.i.d. points in the unit square, chosen with respect to a density $p$. Following the previous section, we will apply this result to densities of the form $p_{L, \alpha}$. In the following 
theorem, the notation w.h.p. (with high probability) refers to a property which holds with probability approaching 1 as $n$ approaches $\infty$.

Theorem 1. Let $p(x, y)$ be a differentiable density distribution on a domain $U$ in the unit square, and let $S$ be a set of $n$ i.i.d. points in the unit square, chosen with respect to the density $p$. Let $A$ and $B$ be points in $U$ with $A \prec B$. Denote by $K=K(A, B)$ the size of the largest increasing subset of $S$, whose points lie between $A$ and $B$. Then the following assertions hold.

1. For all $\varepsilon>0$, w.h.p., $|K-C(A, B) \sqrt{n}|<\varepsilon \sqrt{n}$. Here $C=C(A, B)$ is given by

$$
C_{p, A, B}=\max _{\phi} 2 \int_{x_{A}}^{x_{B}} \sqrt{\phi^{\prime}(x) p(x, \phi(x))} \mathrm{d} x,
$$

where $\phi$ runs through all differentiable, nondecreasing functions on the unit interval, satisfying the boundary conditions $\phi\left(x_{A}\right)=y_{A}$ and $\phi\left(x_{B}\right)=y_{B}$.

2. For any $\varepsilon, \delta>0$, w.h.p., an increasing subset of size $K-\varepsilon \sqrt{n}$ whose elements lie between $A$ and $B$ can be found in a $\delta$-neighborhood of $\phi$ if $\phi$ maximizes the above functional. Here a $\delta$-neighborhood is the set of all points with a vertical distance of less than $\delta$ from some point of the form $(x, \phi(x))$.

\section{The main result}

Let $0<\alpha \leq 1$, and let $\mathscr{D}_{\alpha}$ be the family of all nonincreasing functions from $[0,1]$ to $[\alpha, 1]$. We view the family $\mathcal{D}_{\alpha}$ as representing all back-to-front policies via the upper representation $U=L+\alpha$. Let $I$ be the family of all differentiable, nondecreasing functions from $[0,1]$ to itself. (In the sequel if the upper function is denoted by $U^{*}$ or $\tilde{U}$, say, instead of $U$, then the corresponding lower function is $L^{*}$ or $\tilde{L}$, respectively, etc.) Since the boarding time $T_{U, \alpha, n}$ is given by the size of the longest increasing subsequence, Theorem 1 shows that the boarding time $T$ of $n$ passengers, given a back-to-front airplane policy $U$ and attentiveness parameter $\alpha$, satisfies w.h.p.

$$
-\varepsilon \sqrt{n} \leq T-\mathcal{O}_{U, \alpha} \sqrt{n} \leq \varepsilon \sqrt{n}
$$

for all $\varepsilon>0$, where

$$
\mathcal{O}_{U, \alpha}=\sup _{\psi \in I} 2 \int_{0}^{1} \sqrt{p_{U, \alpha}(z, \psi(z)) \psi^{\prime}(z)} \mathrm{d} z .
$$

The problem of finding an optimal policy is thus reduced to the following problem.

Problem 1. Find a function $U_{\alpha}^{*}$ such that

$$
\mathcal{O}_{U_{\alpha}^{*}, \alpha}=\min _{U \in D_{\alpha}} \mathcal{O}_{U, \alpha}
$$

Given a real number $x$, denote by $\lceil x\rceil$ the least integer which is not smaller than $x$. Our main result is the following.

Theorem 2. For given $0<\alpha \leq 1$, denote

$$
N(\alpha)=\left\lceil\frac{2 / \alpha-3+\sqrt{(2 / \alpha-2)^{2}+1}}{2}\right\rceil \text { and } M(\alpha)=\frac{(N(\alpha)+1) \alpha-1}{N(\alpha)^{2}},
$$


and consider the partition $[0,1]=\bigcup_{i=0}^{N(\alpha)} I_{i}$, where

$$
\begin{aligned}
I_{0} & =\left[0, \frac{M(\alpha)}{\alpha}\right], \\
I_{i} & =\left((2 i-1) \frac{M(\alpha)}{\alpha},(2 i+1) \frac{M(\alpha)}{\alpha}\right], \quad 1 \leq i \leq N(\alpha)-1, \\
I_{N(\alpha)} & =\left((2 N(\alpha)-1) \frac{M(\alpha)}{\alpha}, 1\right] .
\end{aligned}
$$

Then the function $U_{\alpha}^{*}$, defined by

$$
U_{\alpha}^{*}(x)=\frac{i^{2} M(\alpha)}{x}+1-i \alpha, \quad x \in I_{i}, \quad 0 \leq i \leq N(\alpha),
$$

forms a solution of Problem 1. The optimal value of the objective function in (1) is

$$
\mathcal{O}_{\alpha}=\frac{2}{N(\alpha)} \sqrt{N(\alpha)+1-\frac{1}{\alpha}} .
$$

Strictly speaking, the right-hand side of the last formula is undefined for $\alpha=1$. However, it is readily verified that its limit as $\alpha \rightarrow 1-$ is equal to 2 , and we shall take $\mathcal{O}_{1}=2$. This special case, which is of significance as it corresponds to the (non)policy of random order boarding, follows from early results; see [8], [9], [11], and [17].

We remark that the function $U_{\alpha}^{*}$ is composed of a concatenation of hyperbola segments. This fact hints at the Lorentzian interpretation of the problem. Since hyperbolas are the 'circles' of Minkowski space, they can serve as parts of a wave front in the presence of point sources.

It will turn out in the course of the proof that the function $U_{\alpha}^{*}$ in Theorem 2 is not the only solution of Problem 1. For example, if $\alpha \geq \frac{2}{3}$ then the constant rate policy is also optimal; see Subsection 6.2, below. The proof of Theorem 2 implies that the solution $U_{\alpha}^{*}(x)$ is special though, in the sense that it bounds from above all the solutions. In other words, denoting by $\mathcal{O p t} t_{\alpha}$ the set of all solutions of Problem 1, we have

$$
U_{\alpha}^{*}(x)=\sup _{U \in \mathcal{O} p t_{\alpha}} U(x), \quad x \in[0,1] .
$$

However, there are several points at which all solutions of the problem coincide, as Proposition 1 , below, shows.

Proposition 1. Let $0<\alpha \leq 1$, and set $N(\alpha)=\left\lceil\left(2 / \alpha-3+\sqrt{(2 / \alpha-2)^{2}+1}\right) / 2\right\rceil$. For every solution $U \in \mathcal{O p t} t_{\alpha}$, we have

$$
U\left(\frac{i}{N(\alpha)}\right)=1-\frac{i}{N(\alpha)}(1-\alpha), \quad 0 \leq i \leq N(\alpha) .
$$

\section{Proofs}

In this section we fix some $0<\alpha<1$. To prove the main result, we need a few lemmas first.

Lemma 1. Let $U \in \mathcal{D}_{\alpha}$ be an arbitrary fixed function, and let $p=p_{U, \alpha}$. Then

$$
\sup _{\psi \in I} \int_{0}^{1} \sqrt{p(z, \psi(z)) \psi^{\prime}(z)} \mathrm{d} z=\sqrt{\frac{1}{\alpha}} \sqrt{\sup _{0 \leq a<b \leq 1}(b-a)(U(b-)-L(a+))} .
$$


Proof. Set

$$
S=\sup _{\psi \in I} \int_{0}^{1} \sqrt{p(z, \psi(z)) \psi^{\prime}(z)} d z
$$

and

$$
C=\sup _{0 \leq a<b \leq 1}(b-a)(U(b-)-L(a+)) .
$$

First we will show that

$$
S \leq \sqrt{\frac{1}{\alpha}} \sqrt{C} .
$$

Let $\psi_{0} \in I$ be arbitrary and fixed. Set

$$
a_{0}=\sup \left\{x \in[0,1]: \psi_{0}(x)<L(x)\right\} \quad \text { and } \quad b_{0}=\inf \left\{x: \psi_{0}(x)>U(x)\right\}
$$

(where we agree that $a_{0}=0$ if $\psi_{0}(0) \geq L(0)$ and $b_{0}=1$ if $\psi_{0}(1) \leq U(1)$ ).

If $a_{0}=b_{0}$ then $\int_{0}^{1} \sqrt{p\left(z, \psi_{0}(z)\right) \psi_{0}^{\prime}(z)} \mathrm{d} z=0 \leq(\sqrt{1 / \alpha}) \sqrt{C}$. Otherwise we have $a_{0}<b_{0}$. Since $\psi_{0}$ is nondecreasing and $U$ is nonincreasing, $L(x) \leq \psi_{0}(x) \leq U(x)$ throughout the interval $\left(a_{0}, b_{0}\right)$, and therefore

$$
\int_{0}^{1} \sqrt{p\left(z, \psi_{0}(z)\right) \psi_{0}^{\prime}(z)} \mathrm{d} z=\int_{a_{0}}^{b_{0}} \sqrt{\frac{\psi_{0}^{\prime}(z)}{\alpha}} \mathrm{d} z
$$

By Cauchy-Schwarz's inequality we have

$$
\begin{aligned}
\int_{a_{0}}^{b_{0}} \sqrt{\frac{\psi_{0}^{\prime}(z)}{\alpha}} \mathrm{d} z & \leq \sqrt{\int_{a_{0}}^{b_{0}} \psi_{0}^{\prime}(z) \mathrm{d} z \int_{a_{0}}^{b_{0}} \frac{1}{\alpha} \mathrm{d} z} \\
& =\sqrt{\frac{b_{0}-a_{0}}{\alpha}} \sqrt{\psi_{0}\left(b_{0}\right)-\psi_{0}\left(a_{0}\right)}
\end{aligned}
$$

Since

$$
\sqrt{\psi_{0}\left(b_{0}\right)-\psi_{0}\left(a_{0}\right)} \leq \sqrt{U\left(b_{0}-\right)-L\left(a_{0}+\right)}
$$

by (4) and (5), we obtain

$$
\int_{0}^{1} \sqrt{p\left(z, \psi_{0}(z)\right) \psi_{0}^{\prime}(z)} \mathrm{d} z \leq \sqrt{\frac{1}{\alpha}} \sqrt{\left(b_{0}-a_{0}\right)\left(U\left(b_{0}-\right)-L\left(a_{0}+\right)\right)} \leq C,
$$

which implies (3).

Now we will show that

$$
S \geq \sqrt{\frac{1}{\alpha}} \sqrt{C}
$$

Set

$$
\tilde{B}=\{(a, b): 0 \leq a<b \leq 1, U(b-)>L(a+)\} .
$$

If $a$ is a continuity point of $U$, then $(a, b) \in \tilde{B}$ for all $b$ in a sufficiently small right neighborhood of $a$. Since $U$ has at most countably many discontinuities, this implies that $\tilde{B} \neq \varnothing$. Hence,

$$
C=\sup _{(a, b) \in \tilde{B}}(b-a)(U(b-)-L(a+))>0 .
$$


Take an arbitrarily small $\varepsilon>0$. By (7), there exists a pair $\left(a^{*}, b^{*}\right) \in \tilde{B}$ such that

$$
C-\varepsilon \leq\left(b^{*}-a^{*}\right)\left(U\left(b^{*}-\right)-L\left(a^{*}+\right)\right),
$$

or, equivalently,

$$
\sqrt{\frac{C-\varepsilon}{\alpha}} \leq \sqrt{\frac{b^{*}-a^{*}}{\alpha}\left(U\left(b^{*}-\right)-L\left(a^{*}+\right)\right)} .
$$

Let $\tilde{\psi}$ be a linear function on $\left[a^{*}, b^{*}\right]$, with $\tilde{\psi}\left(a^{*}\right)=L\left(a^{*}+\right)$ and $\tilde{\psi}\left(b^{*}\right)=U\left(b^{*}-\right)$. Obviously

$$
\begin{aligned}
\int_{a^{*}}^{b^{*}} \sqrt{p(z, \tilde{\psi}(z)) \tilde{\psi}^{\prime}(z)} \mathrm{d} z & =\int_{a^{*}}^{b^{*}} \sqrt{\frac{1}{\alpha} \frac{\tilde{\psi}\left(b^{*}\right)-\tilde{\psi}\left(a^{*}\right)}{b^{*}-a^{*}}} \mathrm{~d} z \\
& =\sqrt{\frac{b^{*}-a^{*}}{\alpha}\left(U\left(b^{*}-\right)-L\left(a^{*}+\right)\right)} .
\end{aligned}
$$

If $L\left(a^{*}+\right) \neq 0$ and $U\left(b^{*}-\right) \neq 1$, define $\tilde{\psi}$ outside $\left[a^{*}, b^{*}\right]$, in such a way that $\tilde{\psi} \in I$. By (8) and (9), we obtain

$$
S \geq \int_{0}^{1} \sqrt{p(z, \psi \tilde{\psi}(z)) \tilde{\psi}^{\prime}(z)} \mathrm{d} z=\int_{a^{*}}^{b^{*}} \sqrt{p(z, \psi \tilde{\psi}(z)) \tilde{\psi}^{\prime}(z)} \mathrm{d} z \geq \sqrt{\frac{C-\varepsilon}{\alpha}} .
$$

If $L\left(a^{*}+\right)=0$ then, by changing $\tilde{\psi}$ slightly on the interval $\left[a^{*}, a^{*}+\delta\right)$ for an arbitrarily small $\delta>0$, we similarly obtain

$$
S \geq \sqrt{\frac{C-\varepsilon-2 \delta}{\alpha}} .
$$

The case in which $U\left(b^{*}-\right)=1$ leads to (10) in a similar way. Letting $\varepsilon$ and $\delta$ approach 0 , we obtain (6).

The combination of (3) and (6) completes the proof.

In view of Lemma 1, Problem 1 is equivalent to the following problem.

Problem 2. For a given $0<\alpha \leq 1$, find

$$
M=\min _{U \in D_{\alpha}} \sup _{0 \leq a<b \leq 1}(b-a)(U(b-)-L(a+)) .
$$

Lemma 2. If Problem 1 has a solution $U$ then it has a continuous solution $U^{*}$. Moreover, $U^{*}$ may be chosen so that $U^{*}(x) \geq U(x)$ for every $x \in[0,1]$.

Proof. Set

$$
C(U)=\sup _{0 \leq a<b \leq 1}(b-a)(U(b-)-L(a+)), \quad U \in \mathcal{D}_{\alpha} .
$$

Consider an arbitrary function $U_{0} \in \mathscr{D}_{\alpha}$. If $U_{0}$ is discontinuous, we will construct a continuous function $U_{0}^{*} \in \mathscr{D}_{\alpha}$, such that $C\left(U_{0}^{*}\right) \leq C\left(U_{0}\right)$ and $U_{0}^{*}(x) \geq U_{0}(x)$ for $x \in[0,1]$.

Define a function $\tilde{U}_{0}$ by

$$
\tilde{U}_{0}(x)= \begin{cases}U_{0}(0), & x=0 \\ U_{0}(x-), & x>0\end{cases}
$$


Clearly, $\tilde{U}_{0}$ belongs to $\mathscr{D}_{\alpha}$, is continuous from the left, bounds $U_{0}$ from above, and $C\left(\tilde{U}_{0}\right)=$ $C\left(U_{0}\right)$. Hence, to begin with we may assume that $U_{0}$ is continuous from the left.

Let $J_{0}$ denote the (countable) set of all discontinuity points of $U_{0}$. For any $w \in J_{0}$, denote by $j(w)=U_{0}(w)-U_{0}(w+)$ the jump of $U_{0}$ at $w$. Suppose that the maximal jump of $U_{0}$ is at $w_{1}$.

Now we construct a new function $U_{1} \in \mathcal{D}_{\alpha}$, which will be identical with $U_{0}$, except (perhaps) on a small interval $I_{1}=\left[w_{1}, w_{1}+\delta_{1}^{\prime}\right]$, such that $U_{1}$ will be continuous on $I_{1}$ and $C\left(U_{1}\right) \leq C\left(U_{0}\right)$. Take some

$$
0<\delta_{1} \leq \min \left\{\frac{C\left(U_{0}\right) j\left(w_{1}\right)}{1+j\left(w_{1}\right)}, 1-w_{1}\right\}
$$

and set

$$
\begin{gathered}
K_{1}=\frac{U_{0}\left(w_{1}\right)-U_{0}\left(w_{1}+\delta_{1}\right)}{\delta_{1}}, \\
x_{0}=\inf \left\{x \in\left[w_{1}, w_{1}+\delta_{1}\right]: U_{0}\left(w_{1}\right)-K_{1}\left(x-w_{1}\right) \leq U_{0}(x)\right\} .
\end{gathered}
$$

Set

$$
\delta_{1}^{\prime}=x_{0}-w_{1}
$$

Clearly, $0<\delta_{1}^{\prime} \leq \delta_{1}$. Define

$$
U_{1}(x)= \begin{cases}U_{0}\left(w_{1}\right)-K_{1}\left(x-w_{1}\right), & w_{1} \leq x \leq w_{1}+\delta_{1}^{\prime}, \\ U_{0}(x), & \text { otherwise }\end{cases}
$$

The function $U_{1}$ belongs to $\mathscr{D}_{\alpha}$, is continuous from the left, and $U_{1}(x) \geq U_{0}(x)$ throughout $[0,1]$. We want to prove that

$$
C\left(U_{1}\right) \leq C\left(U_{0}\right)
$$

To this end, we have to show that, for any points $x_{1}$ and $x$ with $0 \leq x_{1}<x \leq 1$, we have

$$
\left(x-x_{1}\right)\left(U_{1}(x)-L_{1}\left(x_{1}+\right)\right) \leq C\left(U_{0}\right) .
$$

Set

$$
\Delta x=x-x_{1}, \quad \Delta y=U_{1}(x)-L_{1}\left(x_{1}+\right) .
$$

If both $x_{1}$ and $x$ lie outside the interval $\left[w_{1}, w_{1}+\delta_{1}^{\prime}\right]$, then the left-hand side of (11) remains unchanged if $U_{1}$ and $L_{1}$ are replaced by $U_{0}$ and $L_{0}$, respectively, so that (11) holds. If both of them are within the interval then

$$
\Delta x \Delta y \leq \Delta x \leq \delta_{1}^{\prime} \leq \delta_{1} \leq \frac{C\left(U_{0}\right) j\left(w_{1}\right)}{1+j\left(w_{1}\right)} \leq C\left(U_{0}\right) .
$$

If $w_{1} \leq x_{1} \leq w_{1}+\delta_{1}^{\prime}<x$ then

$$
\Delta x \Delta y \leq \Delta x\left(U_{0}(x)-L_{0}\left(x_{1}+\right)\right) \leq C\left(U_{0}\right) .
$$

It remains to deal with the case in which $x_{1}<w_{1} \leq x \leq w_{1}+\delta_{1}^{\prime}$. Rewrite $\Delta x$ in the form

$$
\Delta x=x-w_{1}+w_{1}-x_{1}=x-w_{1}+\Delta w_{1},
$$


where $\Delta w_{1}=w_{1}-x_{1}$, and rewrite $\Delta y$ in the form

$$
\Delta y=-K_{1}\left(x-w_{1}\right)+\Delta y_{1},
$$

where $\Delta y_{1}=U_{1}\left(w_{1}\right)-L_{1}\left(x_{1}+\right)$.

Now, if $\Delta w_{1} \leq C\left(U_{0}\right)-\delta_{1}^{\prime}$ then

$$
\Delta x \Delta y \leq \Delta x \leq \Delta w_{1}+\delta_{1}^{\prime} \leq C\left(U_{0}\right) .
$$

If $\Delta w_{1}>C\left(U_{0}\right)-\delta_{1}^{\prime}$ then, by (12) and (13),

$$
\Delta x \Delta y=\Delta w_{1} \Delta y_{1}+\left(x-w_{1}\right)\left(\Delta y_{1}-K_{1}\left(x-w_{1}\right)-K_{1} \Delta w_{1}\right) .
$$

Since $0<\delta_{1}^{\prime} \leq \delta_{1} \leq C\left(U_{0}\right) j\left(w_{1}\right) /\left(1+j\left(w_{1}\right)\right)$, we obtain

$$
\frac{\delta_{1}^{\prime}}{j\left(w_{1}\right)} \leq C\left(U^{*}\right)-\delta_{1}^{\prime} .
$$

Note that $K_{1} \geq j\left(w_{1}\right) / \delta_{1}^{\prime}$, and (15) implies that

$$
K_{1} \geq \frac{1}{C\left(U_{0}\right)-\delta_{0}^{\prime}} .
$$

Since $\Delta y_{1} \leq 1$, by (16) this yields

$$
\begin{aligned}
\Delta y_{1}-K_{1}\left(\left(x-w_{1}\right)+\Delta w_{1}\right) & \leq 1-\frac{\left(x-w_{1}\right)+\Delta w_{1}}{C\left(U_{0}\right)-\delta_{1}^{\prime}} \\
& =\frac{C\left(U_{0}\right)-\delta_{1}^{\prime}-\Delta w_{1}-\left(x-w_{1}\right)}{C\left(U_{0}\right)-\delta_{1}^{\prime}} \\
& =\frac{C\left(U_{0}\right)-\delta_{1}^{\prime}-\Delta w_{1}}{C\left(U_{0}\right)-\delta_{1}^{\prime}} \\
& <0 .
\end{aligned}
$$

The last inequality, combined with (14), gives

$$
\Delta x \Delta y \leq \Delta w_{1} \Delta y_{1} \leq C\left(U_{0}\right)
$$

Thus, we have shown that in all cases

$$
C\left(U_{1}\right) \leq C\left(U_{0}\right)
$$

Employing the same process, we construct a sequence of functions $\left(U_{i}\right)_{i=0}^{\infty}$ in $\mathscr{D}_{\alpha}$ such that

$$
U_{0}(x) \leq U_{1}(x) \leq \cdots \leq U_{i}(x) \leq U_{i+1}(x) \leq \cdots, \quad 0 \leq x \leq 1,
$$

and

$$
C\left(U_{0}\right) \geq C\left(U_{1}\right) \geq \cdots \geq C\left(U_{i}\right) \geq C\left(U_{i+1}\right) \geq \cdots .
$$

(Actually, if at some finite stage we obtain a continuous $U_{i}$, we stop the process. In what follows, we shall refer only to the case where each $U_{i}$ is still discontinuous.) 
Each function $U_{i}$ is continuous from the left and agrees (for $i \geq 1$ ) with $U_{i-1}$ except (perhaps) on the interval $I_{i}=\left[w_{i}, w_{i}+\delta_{i}^{\prime}\right]$, where $w_{i}$ is the point of maximal jump of $U_{i-1}$. Moreover, $U_{i}$ is linear and, in particular, continuous on $I_{i}$. We may assume that

$$
I_{i} \cap I_{j}=\varnothing, \quad i \neq j .
$$

By (17), for each $x \in[0,1]$, the sequence $\left(U_{i}\right)_{i=0}^{\infty}$ is eventually constant; hence, the sequence $\left(U_{i}\right)_{i=0}^{\infty}$ converges pointwise to some function $U_{0}^{*}$. Clearly, $U_{0}^{*} \in \mathscr{D}_{\alpha}$ and $U_{0}^{*}(x) \geq U_{0}(x)$ for each $x \in[0,1]$. Let $J_{i}, i \geq 0$, and $J_{0}^{*}$ be the sets of discontinuity points of $U_{i}$ and $U_{0}^{*}(x)$, respectively. Clearly, $J_{0}^{*} \subset J_{i} \subseteq\left\{w \in J_{0}: j(w) \leq j\left(w_{i+1}\right)\right\}$ for each $i$. Since $j\left(w_{i}\right) \rightarrow 0$ as $i \rightarrow \infty$, the function $U_{0}^{*}(x)$ is continuous. Take any two points $x_{1}$ and $x$ with $0 \leq x_{1}<x \leq 1$. For sufficiently large $i$, we have $U_{0}^{*}\left(x_{1}\right)=U_{i}\left(x_{1}\right)$ and $U_{0}^{*}(x)=U_{i}(x)$, and therefore

$$
\left(x-x_{1}\right)\left(U_{0}^{*}(x)-L_{0}^{*}\left(x_{1}\right)\right) \leq C\left(U_{i}\right) \leq C\left(U_{0}\right),
$$

which implies that $C\left(U_{0}^{*}\right) \leq C\left(U_{0}\right)$, and provides the lemma.

For $0<\alpha \leq 1$ and $M>0$, set

$$
\mathscr{D}_{\alpha, M}=\left\{U \in \mathscr{D}_{\alpha}: \sup _{0 \leq a<b \leq 1}(U(b-)-L(a+))(b-a) \leq M\right\} .
$$

Similarly to Lemma 2, we can prove the following lemma.

Lemma 3. If $\mathcal{D}_{\alpha, M} \neq \varnothing$ for given $0<\alpha \leq 1$ and $M>0$ then, for any function $U \in D_{\alpha, M}$, there exists a continuous function $U^{*} \in \mathscr{D}_{\alpha, M}$ such that $U^{*}(x) \geq U(x)$ for $x \in[0,1]$.

Let $\mathcal{C}_{M}$ be the family of all nonincreasing, continuous functions $U:[0,1] \longrightarrow(-\infty, 1]$, satisfying

$$
\sup _{0 \leq a<b \leq 1}(U(b)-L(a))(b-a)=M, \quad M>0 .
$$

Lemma 4. Suppose that $\mathcal{C}_{M} \neq \varnothing$ for a given $M>0$. Let

$$
U_{0}(x)=\sup _{U \in \mathcal{C}_{M}} U(x), \quad x \in[0,1]
$$

Then $U_{0}$ is nonincreasing and satisfies

$$
\left(U_{0}(b-)-L_{0}(a+)\right)(b-a) \leq M, \quad 0 \leq a<b \leq 1 .
$$

Proof. Clearly, $U_{0}$ is nonincreasing. For arbitrary fixed $\delta>0$, we have

$$
\begin{aligned}
& \left(U_{0}(b-)-L_{0}(a+)\right)(b-a) \\
& \quad \leq \frac{b-a}{b-a-2 \delta}((b-\delta)-(a+\delta))\left(U_{0}(b-\delta)-L_{0}(a+\delta)\right) .
\end{aligned}
$$

For arbitrary fixed $\varepsilon>0$, select a function $U^{*} \in \mathcal{C}_{M}$ such that

$$
U_{0}(b-\delta) \leq U^{*}(b-\delta)+\varepsilon .
$$

Since $L^{*}(a+\delta) \leq L_{0}(a+\delta)$, we obtain

$$
\begin{aligned}
& ((b-\delta)-(a+\delta))\left(U_{0}(b-\delta)-L_{0}(a+\delta)\right) \\
& \quad \leq((b-\delta)-(a+\delta))\left(U^{*}(b-\delta)+\varepsilon-L^{*}(a+\delta)\right) \\
& \quad \leq M+\varepsilon .
\end{aligned}
$$


Substituting in (19), we find that

$$
\left(U_{0}(b-)-L_{0}(a+)\right)(b-a) \leq \frac{b-a}{b-a-2 \delta}(M+\varepsilon) .
$$

Letting $\delta \rightarrow 0$ and $\varepsilon \rightarrow 0$, we obtain (18), which completes the proof.

For given $0<M \leq \alpha \leq 1$, set $N=\lceil(\alpha / M-1) / 2\rceil$, and consider the partition $[0,1]=$ $\bigcup_{i=0}^{N} J_{i}$, where

$$
\begin{aligned}
J_{0} & =\left[0, \frac{M}{\alpha}\right], \\
J_{i} & =\left((2 i-1) \frac{M}{\alpha},(2 i+1) \frac{M}{\alpha}\right], \quad 1 \leq i \leq N-1, \\
J_{N} & =\left((2 N-1) \frac{M}{\alpha}, 1\right] .
\end{aligned}
$$

Proposition 2. Set

$$
U^{*}(x)=\frac{i^{2} M}{x}+1-i \alpha, \quad x \in J_{i}, \quad 0 \leq i \leq N .
$$

Then

(i) $U^{*} \in \mathcal{C}_{M}$;

(ii) $U^{*}(x)=\sup _{U \in \mathfrak{C}_{M}} U(x), x \in[0,1]$.

Proof. (i) It is easy to verify that $U^{*}$ is nonincreasing, continuous, and bounded above by 1 . It remains to show that

$$
\sup _{0 \leq a<b \leq 1}\left(U^{*}(b)-L^{*}(a)\right)(b-a)=M .
$$

Let $0 \leq i \leq N$, and let $b \in J_{i}$ be an arbitrary fixed point of $J_{i}$. We shall split the proof into four cases.

If $0 \leq i \leq 2$ and $a \in I_{0}$ then

$$
\begin{aligned}
\left(U^{*}(b)-L^{*}(a)\right)(b-a) & =\left(\frac{i^{2} M}{b}-(i-1) \alpha\right)(b-a) \\
& \leq i^{2} M-(i-1) \alpha b \\
& \leq M .
\end{aligned}
$$

For any $0 \leq i \leq N$, if $b-M / \alpha<a<b$ then

$$
\left(U^{*}(b)-L^{*}(a)\right)(b-a)=\left(U^{*}(b)-U^{*}(a)+\alpha\right)(b-a)<\alpha \frac{M}{\alpha}=M .
$$

If $i \geq 3$ and $a \in J_{k}$ for some $0 \leq k \leq i-3$ then $0 \leq a \leq(2 i-5)(M / \alpha)$, and therefore

$$
U^{*}(b)-L^{*}(a) \leq \frac{i^{2} M}{(2 i-1)(M / \alpha)}-\frac{(i-3)^{2} M}{(2 i-5)(M / \alpha)}-2 \alpha=-\frac{\alpha}{(2 i-1)(2 i-5)}<0 .
$$


Finally, let $i \geq 1$ and $a \in J_{k}$ for some $\max (1, i-2) \leq k \leq i$. Set

$$
A=M i^{2}-\alpha b(i-k-1)
$$

and

$$
d=\frac{b-a}{M / \alpha} .
$$

Define a quadratic function $h$ by

$$
h(z)=-M A z^{2}+\alpha b\left(A-M\left(k^{2}-1\right)\right) z-\alpha^{2} b^{2} .
$$

A routine calculation gives

$$
\left(U^{*}(b)-L^{*}(a)\right)(b-a)-M=\frac{M}{\alpha^{2} a b} h(d) .
$$

It is easy to verify that $A>0$ and that the discriminant of $h$ satisfies

$$
\Delta=\alpha^{2} b^{2}\left(A-M(k+1)^{2}\right)\left(A-M(k-1)^{2}\right) \leq 0 .
$$

It follows that $h(d) \leq 0$, which implies that

$$
\left(U^{*}(b)-L^{*}(a)\right)(b-a) \leq M .
$$

Altogether, by (21), (22), (23), and (24), we obtain

$$
\left(U^{*}(b)-L^{*}(a)\right)(b-a) \leq M, \quad 0 \leq a \leq b \leq 1 .
$$

Conversely, let $b \in J_{i}$ for any $1 \leq i \leq N$, and set $a_{0}=((i-1) / i) b$. It is easy to verify that

$$
\left(U^{*}(b)-L^{*}\left(a_{0}\right)\right)\left(b-a_{0}\right)=M, \quad b \in J_{i}, 1 \leq i \leq N,
$$

which, combined with (25), completes the proof of part (i).

(ii) Set

$$
U_{0}(x)=\sup _{U \in \mathcal{C}_{M}} U(x), \quad x \in[0,1] .
$$

Suppose that $U_{0}$ is not identical with $U^{*}$. Let $j$ be the smallest index $i$ for which the restrictions of $U_{0}$ and $U^{*}$ to $J_{i}$ are nonidentical. Clearly, $1 \leq j \leq N$ and $U_{0}(x)=U^{*}(x)$ for each point $x \in J_{j-1}$. Let $b_{0} \in J_{j}$ be a point with $U_{0}\left(b_{0}\right)>U^{*}\left(b_{0}\right)$. Set $a_{0}=((j-1) / j) b_{0}$. Then $a_{0} \in J_{j-1}$ and $U_{0}\left(a_{0}\right)=U^{*}\left(a_{0}\right)$. As in (26),

$$
\left(U_{0}\left(b_{0}\right)-L_{0}\left(a_{0}\right)\right)\left(b_{0}-a_{0}\right)>\left(U^{*}\left(b_{0}\right)-L^{*}\left(a_{0}\right)\right)\left(b_{0}-a_{0}\right)=M,
$$

which contradicts Lemma 4, and thus completes the proof of part (ii).

Proof of Theorem 2. If $N=N(\alpha)$ and $M=M(\alpha)$, then $U^{*}(1)=\alpha$, where $U^{*}$ is defined as in (20). By part (i) of Proposition 2 we have $U^{*} \in \mathcal{C}_{M}$, and thus $U^{*} \in \mathscr{D}_{\alpha}$. Hence, $U^{*} \in \mathcal{D}_{\alpha, M}$.

Let $0<M<((N+1) \alpha-1) / N^{2}$ be arbitrary and fixed. Suppose that there exists some $\tilde{U} \in \mathcal{D}_{\alpha, M}$. By Lemma 3 , there exists a continuous function $\tilde{U}_{0} \in \mathcal{D}_{\alpha, M}$. Since, by part (ii) 
of Proposition 2, $U^{*}$ is the supremum of all continuous functions $U \in \mathcal{C}_{M}$, and it is easy to verify that

$$
U^{*}(1)=N^{2} M+1-N \alpha<(N+1) \alpha-N \alpha=\alpha,
$$

it follows that, for any $U \in \mathcal{C}_{M}$, we have $U(1)<\alpha$. Thus, for any $U \in \mathcal{C}_{M}$, we obtain $U \notin \mathscr{D}_{\alpha, M}$, which implies that $\mathscr{D}_{\alpha, M}=\varnothing$.

Hence, $U^{*}$, with $M=((N+1) \alpha-1) / N^{2}$, forms a solution of Problem 1. In view of Lemma 1 , the optimal value is

$$
\mathcal{O}_{\alpha}=\left(\frac{2}{N}\right) \sqrt{N+1-1 / \alpha}
$$

Proof of Proposition 1. Let $U \in \mathcal{O} p t$, and let $N=N(\alpha)$. First note that $i / N \in I_{i}$ for $i=0,1, \ldots, N$. We start by examining the value of $U$ at the last of the points $i / N$, namely $1 \in I_{N}$. On the one hand, $U(1)=L(1)+\alpha \geq \alpha$, and on the other hand, by Proposition 2(ii) and Lemma 2, we have $U(1) \leq U^{*}(1)=\alpha$. Thus, every $U \in \mathcal{O} p t$ satisfies $U(1)=U^{*}(1)=\alpha$. Next consider the point $(N-1) / N \in I_{N-1}$. Since $U$ is a solution of Problem 2 with optimal value $M=((N+1) \alpha-1) / N^{2}$, we have

$$
\left(U(1)-L\left(\frac{N-1}{N}\right)\right)\left(1-\frac{N-1}{N}\right) \leq\left(U(1-)-L\left(\frac{N-1}{N}+\right)\right) \frac{1}{N} \leq M .
$$

On the other hand, $U^{*}$ also provides the same optimal value $M$ of Problem 2, and Proposition 2(ii) and Lemma 2 imply that

$$
L\left(\frac{N-1}{N}\right) \leq L^{*}\left(\frac{N-1}{N}\right) .
$$

Thus,

$$
\left(U(1)-L\left(\frac{N-1}{N}\right)\right) \frac{1}{N} \geq\left(U^{*}(1)-L^{*}\left(\frac{N-1}{N}\right)\right) \frac{1}{N}=M,
$$

which implies that,

$$
U\left(\frac{N-1}{N}\right)=U^{*}\left(\frac{N-1}{N}\right)=1-\frac{N-1}{N}(1-\alpha) .
$$

Now suppose that, for some $1 \leq i \leq N$, we have proved that $U(i / N)=1-(i / N)(1-\alpha)$. We can easily check that

$$
\left(U^{*}\left(\frac{i}{N}\right)-L^{*}\left(\frac{i-1}{N}\right)\right) \frac{1}{N}=M, \quad 1 \leq i \leq N,
$$

which, in the same way as above, implies that

$$
U\left(\frac{i-1}{N}\right)=1-\left(\frac{i-1}{N}\right)(1-\alpha) .
$$

This completes the proof. 


\section{Examples}

\subsection{Jump policies}

A jump policy is a back-to-front policy, where the function $L(x)$ is of the form $L(x)=t_{i}$ for $r_{i} \leq x \leq r_{i+1}$, for some constants $0=t_{0}<t_{1}<t_{2}<\cdots<t_{k+1}=1$ and $0=r_{0}<$ $r_{1}<\cdots<r_{k+1}=1$, for $i=1, \ldots, k$. The parameter $k$ denotes the number of jumps in the policy, and the $i$ th jump allows the group of passengers from rows $r_{i}$ to $r_{i+1}$ to join the queue at time $t_{k-i}$. The idea is to allow the passengers from the $k$ th (last) group to board first, followed by the passengers from the next to last group, and so on. This will occur if $\alpha \leq \min _{i}\left(t_{i-1}-t_{i}\right)$, an inequality which we assume. Under such circumstances, the boarding time will in fact be independent of the differences $t_{i-1}-t_{i}$, since the restrictions on the order of passengers in the queue are independent of the differences, and we may assume that $t_{i}=1-i / k$ and that $\alpha=1 / k$. Applying Theorem 1, we see that the boarding time (after division by $\sqrt{n}$ ) is asymptotically $\max _{i} 2 \sqrt{r_{i+1}-r_{i}}$. The boarding time is minimized by choosing all jumps (groups) to be of equal size, in which case we obtain an asymptotic boarding time of $2 \sqrt{1 / k}=2 \sqrt{\alpha}$.

\subsection{Constant rate policies}

The constant rate policy with parameter $\alpha$ is given by the affine function $L_{\alpha}(x)=$ $(1-\alpha)(1-x)$. As noted in the introduction, such policies allow passengers from new rows to join the boarding queue at a constant rate, which is adjusted to the attentiveness of the passengers. We know, by Lemma 1 , that the normalized boarding time $\mathcal{O}_{L_{\alpha}}$ is given by $\max _{0 \leq a<b \leq 1} 2 \sqrt{(b-a)\left(U_{\alpha}(b)-L_{\alpha}(a)\right)}$. By elementary computations we have

$$
\mathcal{O}_{L_{\alpha}}=2 \sqrt{\frac{\alpha}{4(1-\alpha)}}=\sqrt{\frac{1}{1-\alpha}} \sqrt{\alpha}, \quad \alpha \leq \frac{2}{3},
$$

while

$$
\mathcal{O}_{L_{\alpha}}=2 \sqrt{2-\frac{1}{\alpha}}, \quad \alpha \geq \frac{2}{3} .
$$

A comparison with jump policies shows that constant rate policies are preferable to jump policies. We now compare constant rate policies to the optimal policies. When $\alpha \geq \frac{2}{3}$, the boarding time coincides with that of the optimal policy and, hence, the constant rate policy is optimal for $\alpha \geq \frac{2}{3}$. For example, for $\alpha=\frac{7}{9}$, the constant rate policy, $L_{\alpha}(x)=\frac{2}{9}-\frac{2}{9} x$, $0 \leq x \leq 1$, and the optimal policy, defined in Theorem 2 ,

$$
L_{\alpha}^{*}(x)=U_{\alpha}^{*}(x)-\alpha= \begin{cases}\frac{2}{9}, & 0 \leq x \leq \frac{5}{7}, \\ \frac{5}{9 x}-\frac{5}{9}, & \frac{5}{7}<x \leq 1,\end{cases}
$$

provide the same normalized boarding time $2 \sqrt{5 / 7}$. As mentioned above, the optimal policies are not unique, and Proposition 1 identifies an $\alpha$-dependent discrete substructure, common to all optimal solutions. However, the optimal policy (2), defined in Theorem 2 is special, in the sense that it bounds from above all the optimal solutions. For $\alpha<\frac{2}{3}$, the constant rate policy is rarely optimal, but we show that it is worse by at most a factor of $3 / 2 \sqrt{2} \approx 1.06$ than the optimal policy for all values of $\alpha$. Let $R(\alpha)$ denote the ratio between the performance of the optimal policy and that of the constant rate policy. We are interested in the maximal value 
of $1 / R$. We have $R(\alpha)=1$ for $\alpha \geq \frac{2}{3}$. For $\alpha \leq \frac{2}{3}$, we have

$$
R(\alpha)=\frac{2}{N(\alpha)} \sqrt{\frac{((N(\alpha)+1) \alpha-1)(1-\alpha)}{\alpha^{2}}} .
$$

$N(\alpha)$ is an integer-valued function, which assumes the value $N \geq 1$ in the range $\alpha_{N-1} \geq \alpha>$ $\alpha_{N}$, where

$$
\alpha_{N}=\frac{2 N+1}{N^{2}+3 N+1} \quad \text { for } N>0
$$

and $\alpha_{0}=1$. Fixing some $N$, it is easy to see that the function

$$
R_{N}(\alpha)=\frac{2}{N} \sqrt{\frac{((N+1) \alpha-1)(1-\alpha)}{\alpha^{2}}}
$$

has a single maximal value in the range $\alpha>0$, which is at the point $\beta_{N}=2 /(N+2)$. A simple computation shows that $\beta_{N}$ satisfies $\alpha_{N-1}>\beta_{N}>\alpha_{N}$ and that $R\left(\beta_{N}\right)=1$. Hence, the constant rate policy is optimal for each $\alpha=\beta_{N}$, and only at these values. We also conclude that the minimal value of $R$ in the range $\alpha_{N-1} \geq \alpha>\alpha_{N}$ must lie either at $\alpha_{N-1}$ or at $\alpha_{N}$. Substituting the formula for $\alpha_{N}$ into $R$, we obtain a sequence of values

$$
\gamma_{N}=R\left(\alpha_{N}\right)=\frac{\sqrt{(N+1 / 2)^{2}-1 / 4}}{2(N+1 / 2)} .
$$

It is clear that $\gamma_{N}$ is an increasing sequence, and hence the minimal value is $\gamma_{1}=2 \sqrt{2} / 3$, as required.

\section{Acknowledgements}

The authors express their gratitude to Ofer Zeitouni for several useful conversations, and an anonymous referee of the paper for the detailed reading of the manuscript and the enlightening comments.

\section{References}

[1] Aldous, D. And Diaconis, P. (1999). Longest increasing subsequences: from patience sorting to the BaikDeift-Johansson theorem. Bull. Amer. Math. Soc. 36, 413-432.

[2] Arias-Castro, E., Donoho, D. L., Huo, X. And Tovey, C. A. (2005). Connect-the-dots: how many random points can a regular curve pass through? Adv. Appl. Prob. 37, 571-603. (Correction: 38 (2006), 579).

[3] Bachmat, E. (2007). Discrete space-time and its applications. In Random Matrices, Integrable Systems and Applications, eds J. Baik et al., American Mathematical Society, Providence, RI.

[4] BАснмат, E. (2007). Average case analysis of disk scheduling, increasing subsequences and spacetime geometry. Algorithmica 49, 212-231.

[5] Bachmat, E. ANd ElKin M. (2007). Bounds on the performance of back-to-front boarding policies. Submitted.

[6] Bachmat, E. et al. (2005). Analysis of airplane boarding times. Submitted.

[7] Bachmat, E. et al. (2006). Analysis of airplane boarding via space-time geometry and random matrix theory. J. Physics A Math. General 39, L453-L459.

[8] Baik, J., Deift, P. And Johansson, K. (1999). On the distribution of the length of the longest increasing subsequence of random permutation. J. Amer. Math. Soc. 12, 1119-1178.

[9] Deuschel, J. D. and Zeitouni, O. (1995). Limiting curves for i.i.d. records. Ann. Prob. 23, 852-878.

[10] Ferrari, P. AND NAgel, K. (2005). Robustness of efficient passenger boarding in airplanes. Transportation Res. Board Ann. Meeting, paper number 05-0405, Washington D.C. Available at http://gulliver.trb.org/ am/ip/assembly_detail.asp?id=3705.

[11] Logan, B. F. AND Shepp, A. (1977). A variational problem for random Young tableaux. Adv. Math. 26, $206-222$. 
[12] Marelli, S., Mattocks, G. And Merry, R. (1998). The role of computer simulation in reducing airplane turn time. Boeing Aero Magazine 1. Available at http://www.boeing.com/commercial/aeromagazine/ aero_01/index 2. html

[13] Prahofer, M. and Spohn, H. (2000). Universal distributions for growth processes in $1+1$ dimensions and random matrices. Physical Rev. Lett. 84, 4882-4885.

[14] Van den Briel, M., Villalobos, J. and Hogg, G. (2003). The aircraft boarding problem. Proc. 12th Industrial Eng. Res. Conf. IERC, CD ROM, paper number 2153.

[15] VAn DEN Briel, M. et al. (2005). America West develops efficient boarding strategies. Interfaces 35, 191-201.

[16] Van Landeghem, H. and Beuselinck, A. (2002). Reducing passenger boarding time in airplanes: a simulation approach. Europ. J. Operat. Res. 142, 294-308.

[17] Vershik, A. AND Kerov, S. (1977). Asymptotics of the Plancherel measure of the symmetric group and the limit form of Young tableaux. Soviet Math. Dokl. 18, 527-531. 